\title{
52
}

\section{Aircraft trajectory optimization using nonlinear programming}

\author{
T. Raivio, H. Ehtamo, R. P. Hämäläinen \\ Systems Analysis Laboratory, Helsinki University of Technology \\ Otakaari 1 M, 02150 Espoo, Finland. Tel. +358-0-4513059. \\ Fax: +358-0-451 3096. e-mail: tuomas.raivio@hut.fi
}

\begin{abstract}
We describe two discretization methods, direct collocation and a scheme based on differential inclusion, that enable the solution of optimal control problems by nonlinear programming. We apply the methods in calculating optimal trajectories for a modern fighter aircraft. Unlike collocation, the differential inclusion scheme converges robustly even in the presence of singular controls.
\end{abstract}

\section{Keywords}

Trajectory optimization, direct methods, discretization, nonlinear programming, singular controls.

\section{INTRODUCTION}

Aircraft trajectory optimization problems constitute a challenging class of optimal control problems. A six degrees of freedom aircraft model consists of 12 first order differential equations. In trajectory optimization the equations of rotation can be neglected, but the remaining model still includes six nonlinear first order equations of motion (see, e.g. , Miele (1962)).

The optimal control is usually solved either by indirect or direct methods. Indirect methods solve the multipoint boundary value problem arising from the necessary conditions for optimality. The methods provide accurate results but require a good initial guess. This stems mainly from the nonlinear and unstable nature of the boundary value problem and Newton-type solution methods.

Infinite dimensional direct methods attempt to overcome these difficulties. They improve a given nominal solution by a gradient search in a function space. A larger convergence domain is often achieved, but other difficulties, like the treatment of constraints, arise.

In many cases the accuracy of the solution is not as important as is the convergence of the solution method. The convergence could be improved by replacing the original infinitedimensional problem with a finite-dimensional approximation, in which the differential 
equation constraint is satisfied only pointwise. Restricting to a finite dimension also allows the use of ordinary nonlinear optimization.

The discretization of the problem can be carried out in a number of ways. Hargraves et al. (1981) present the state trajectories with high-order patched polynomials, whose coefficients are the decision variables. Betts and Huffman (1993) discuss trapetzoidal, HermiteSimpson and Runge-Kutta discretization. In the following we describe two schemes, direct collocation and a recently proposed method based on differential inclusions Seywald (1994). In the former approach the states and controls are approximated by piecewisely defined low-order polynomials. The latter scheme makes use of differential inclusion (see, e.g. , Aubin and Frankowska (1989)) and the concept of attainability. We demonstrate and compare the performance of these methods and the accuracy of the results. The numerical examples involve also singular controls.

\section{AIRCRAFT MODEL}

The dynamics of a point mass aircraft can be described by the following system of equations (see, e.g. , Miele (1962)):

$$
\begin{aligned}
\dot{x} & =v \cos \gamma \cos \chi \\
\dot{y} & =v \cos \gamma \sin \chi \\
\dot{h} & =v \sin \gamma \\
\dot{\gamma} & =\frac{g}{v}(n \cos \mu-\cos \gamma) \\
\dot{\chi} & =\frac{g}{v} \frac{n \sin \mu}{\cos \gamma} \\
\dot{v} & =\frac{1}{m}\left[u T_{\max }(h, M(h, v))-D(h, v, M(h, v), n)\right]-g \sin \gamma
\end{aligned}
$$

The state variables $x, y, h, v, \gamma$ and $\chi$ are the $x$ and the $y$ coordinates, altitude, velocity, flight path angle and heading angle of the aircraft, respectively. The acceleration due to gravity, $g$, is assumed constant. $T_{\max }(h, M(h, v))$ denotes the maximum available thrust force, $u$ the throttle setting, $D(\cdot)$ the drag force and $M(\cdot)$ the Mach number. For short flight times the mass of the aircraft can be assumed constant.

The normal acceleration of the aircraft is controlled with the normal load factor $n$ and the tangential acceleration with the throttle setting $u \in[0,1]$. To produce a horizontal turn, the normal load factor can be directed away from the vertical plane with the bank angle $\mu \in[-\pi, \pi[$.

The load factor $n$ cannot be chosen freely. At low velocities, a large load factor requires a large angle of attack which results in loss of lift force and stall. At higher velocities, the magnitude of the load factor is constrained by the largest acceleration that the pilot and the aircraft withstand. Here the smallest allowed load factor is set to zero to ensure the uniqueness of the control variable combinations.

The aircraft drag is assumed to obey a shifted quadratic polar emerging from the actively controlled aircraft wing Ehtamo et al. (1994):

$$
C_{D}(M(h, v), n)=C_{D_{0}}(M(h, v))+K(M(h, v))\left(C_{L}(\cdot)-a\right)^{2}, a>0 .
$$


Here $C_{D}(\cdot), C_{D_{0}}(\cdot)$ and $K(\cdot)$ denote the total, zero-lift and induced drag coefficients, respectively. The drag force becomes

$$
\begin{aligned}
& D(h, v, M(h, v), n)=C_{D}(M(h, v), n) S q(h, v)= \\
& \left(C_{D_{0}}(M(h, v))+K(M(h, v))\left(\frac{n m g}{S q(h, v)}-a\right)^{2}\right) S q(h, v), \quad q(h, v)=\frac{1}{2} \varrho(h) v^{2} .
\end{aligned}
$$

$S$ and $q(h, v)$ stand for the reference wing area and the dynamic pressure. The coefficients $C_{D_{0}}(M(\cdot))$ and $K(M(\cdot))$ are approximated by rational polynomials on the basis of realistic tabular data. The maximum thrust data is approximated by a two-dimensional polynomial. The air density and the speed of the sound are taken from the standard ISA atmosphere.

\section{THE DISCRETIZATION METHODS}

\section{Direct collocation}

In direct collocation the state trajectories and admissible controls are constrained to lie in the space of piecewise polynomials of time with given degree. The polynomials must satisfy the state equation in a finite number of points at each interval. In this paper the state trajectories are interpolated by Hermite interpolation and cubic polynomials. The controls are approximated piecewise linearly. The slope of the approximating polynomial must coincide with the state equation value at the middle of each discretization interval. The optimal values of the control and state variables in the discretization points are selected through nonlinear optimization to minimize the cost function. The method or its variants have been applied to various trajectory optimization tasks (see, e.g. , Hargraves and Paris (1987)) but also to facilitate the solving of complex pursuit-evasion games by providing an initial guess for the solution Lachner et al. (1994).

Differential inclusion scheme

Another way to discretize the problem is to require that each subsequent state can be attained from the preceding state. Given $t_{0}$, an initial state vector $x\left(t_{0}\right)=x_{0}$ and $t_{1}$, the set of attainability $K\left(x_{0}, t_{0}, t_{1}\right)$ is defined as the collection of the states that can be reached from $x_{0}$ in $\left[t_{0}, t_{1}\right]$ using admissible controls $u(t), t \in\left[t_{0}, t_{1}\right]$ (Lee and Markus, 1986). In general, this set cannot be expressed explicitly. To approximate it we use the set of attainable state rates at state $x(t)$ defined by

$\mathcal{H}(x(t))=\left\{\dot{x}(t) \in R^{n} \mid \dot{x}(t)=f(x(t), u(t)), u(t)\right.$ admissible $\}$

Here $f(\cdot)$ refers to the RHS of the state equations. In the following, we drop the argument $t$ for clarity. The set $\mathcal{H}(x)$ is sometimes called the hodograph of the system (e.g. Seywald (1994)). We next assume that $u$ can be eliminated from the definition of $\mathcal{H}(x)$. That is, there exist smooth functions $p: R^{n} \times R^{n} \mapsto R^{p}$ and $q: R^{n} \times R^{n} \mapsto R^{q}$ such that $\mathcal{H}(x)$ can be expressed as

$\mathcal{H}(x)=\left\{\dot{x} \in R^{n} \mid p(\dot{x}, x)=0, q(\dot{x}, x) \leq 0\right\}$. 
The existence of $p$ and $q$ depends on the problem considered. In practice, they are derived by eliminating the controls from the state equations and then using the control constraints.

The first order approximation of the set of attainability is

$\hat{K}(x, t, t+\Delta t)=\left\{y \in R^{n} \mid y=x+\Delta t \cdot \mathcal{H}(x)\right\}$

where $\Delta t \cdot \mathcal{H}(x)=\{\Delta t \cdot \dot{x} \mid \dot{x} \in \mathcal{H}(x)\}$. The condition that takes the system dynamics into account is that each subsequent discretized state must lie in the approximated set of attainability of its predecessor.

Two remarks are to be made. First, in this form the differential inclusion scheme is merely a first order discretization of the system. Nevertheless, the elimination of the control variables is expected to be computationally beneficial, especially in optimization problems that include singular controls. Second, the system equations must be invertible with respect to the control variables.

\section{NUMERICAL EXAMPLES}

In the first numerical example the task is to find a minimum time trajectory from the initial conditions

$$
\begin{array}{ll}
x(0)=0 \mathrm{~m}, & y(0)=0 \mathrm{~m}, \quad h(0)=2,000 \mathrm{~m}, \\
v(0)=200 \mathrm{~m} / \mathrm{s}, & \gamma(0)=0 \mathrm{rad}, \quad \chi(0)=0 \mathrm{rad}
\end{array}
$$

to the point $(x(T)=10,000 \mathrm{~m}, y(T)=15,000 \mathrm{~m}, h(T)=7,000 \mathrm{~m})$, final heading $\chi(T)=$ $0.35 \mathrm{rad}$ and to level flight, $\gamma(T)=0 \mathrm{rad}$. The mass of the aircraft was set to $10,000 \mathrm{~kg}$. The problem was solved using Sequential Quadratic Programming and 5, 10,15 and 20 equidistant discretization points. A representative solution of both methods, together with a reference solution obtained by multiple shooting, is presented in fig. 1. The iteration results are summarized in table 1.

With differential inclusion the errors decrease rapidly when the amount of discretization points is increased. In collocation, the situation is opposite: in our case five nodes provided the best result. The reason is probably that with short discretization intervals the best approximation approaches a line segment, which turns the collocation constraints more linearly dependent.

The dynamic pressure constraint $q(h, v)-q_{\max } \leq 0$ in minimum time problems is known to lead to first order singularity on the controls (see, e.g., Seywald et al. (1994)). To compare the convergence properties of the methods when singular controls arise, a minimum time descent was computed from the initial conditions

$$
\begin{array}{lll}
x(0)=0 \mathrm{~m}, & y(0)=0 \mathrm{~m}, & h(0)=5,000 \mathrm{~m}, \\
v(0)=300 \mathrm{~m} / \mathrm{s}, & \gamma(0)=0 \mathrm{rad}, & \chi(0)=0 \mathrm{rad}
\end{array}
$$

to the point $x(T)=20,000 \mathrm{~m}, y(T)=10,000 \mathrm{~m}$ and $h(T)=1,000 \mathrm{~m}$, with free final flight path angle and heading with the condition

$q(h, v) \leq 80,000 N / m^{2}$. 


\begin{tabular}{lcccccccc}
\hline & \multicolumn{3}{c}{ Direct collocation } & \multicolumn{3}{c}{ Differential inclusion } \\
No. of nodes & 5 & 10 & 15 & 20 & 5 & 10 & 15 & 20 \\
\hline Final time & 59.6 & 61.6 & 60.3 & 61.2 & 60.0 & 59.2 & 59.3 & 59.4 \\
$\left(T_{\text {ref }}=60.2 \mathrm{~s}\right)$ & & & & & & & & \\
Dimension & 41 & 81 & 121 & 161 & 31 & 61 & 91 & 121 \\
Simple bounds & 15 & 30 & 45 & 60 & 5 & 10 & 15 & 20 \\
Constraints & 35 & 65 & 95 & 125 & 45 & 85 & 125 & 165 \\
Major iterations & 30 & 19 & 18 & 21 & 18 & 31 & 45 & 55 \\
Minor iterations & 83 & 74 & 51 & 60 & 53 & 158 & 296 & 1099 \\
\hline
\end{tabular}

Table 1 Summary of the iteration characteristics. 'Simple bounds' means the amount of decision variable bounds, whereas 'constraints' refers to the number of true constraints. 'Major iterations' means the amount of QP problems solved and 'minor iterations' the cumulative amount of iterations needed to solve them.
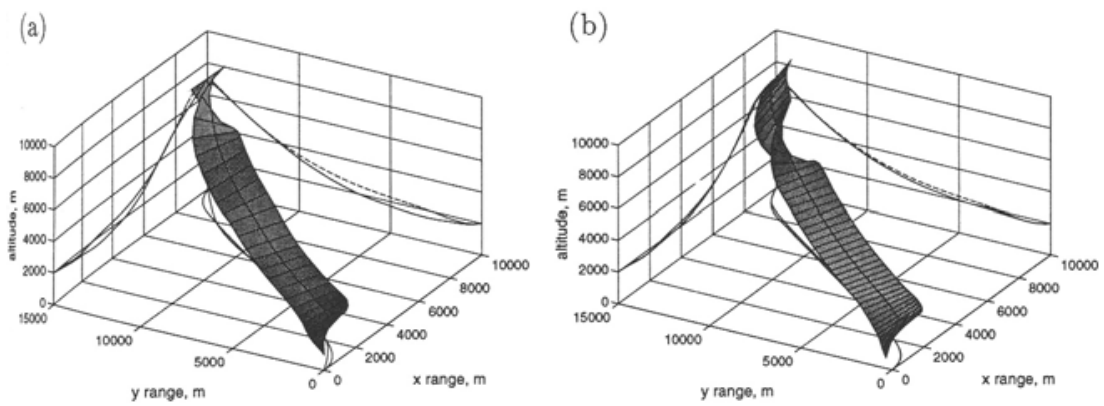

Figure 1 The solution obtained with (a) direct collocation and 5 nodes and (b) differential inclusion and 15 nodes. The stripe describes the bank angle of the aircraft. The dashed projections represent the reference solution obtained with multiple shooting.

In an unconstrained solution the maximum pressure would be over $120,000 \mathrm{~N} / \mathrm{m}^{2}$, which is untolerable for most aircraft.

Six nodes were employed in the collocation and 15 nodes in the differential inclusion. The solution trajectories are presented in fig. 2 . The state and control variables are presented in fig. 3 . The pressure constraint becomes active at $t \cong 27 \mathrm{~s}$. After that the controls turn singular. The constraint remains active for the rest of the trajectory. Note that the singularity of the controls cannot be deduced without some additional knowledge on the problem.

Singular controls shrunk the convergence domain of collocation for convergence was obtained only after numerous attempts with different initial guesses. The differential inclusion scheme did not exhibit such convergence problems, as expected. 

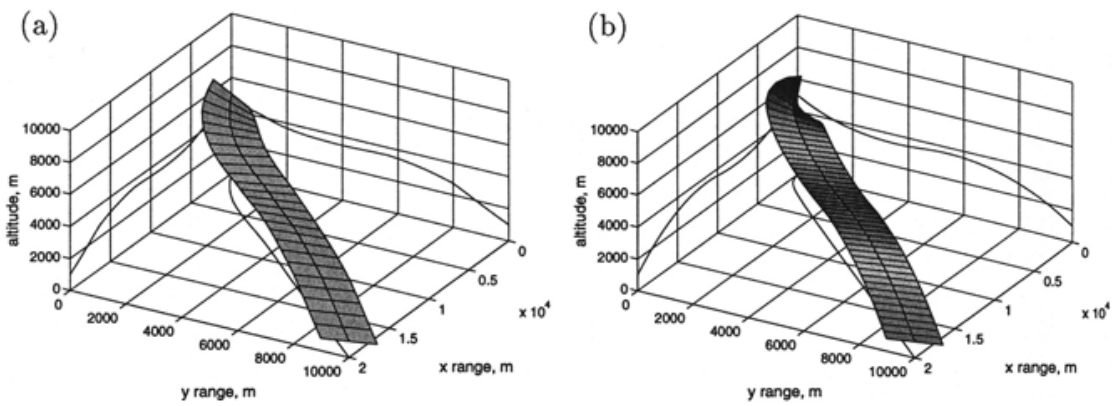

Figure 2 The descent trajectories obtained with (a) collocation and 6 nodes and (b) differential inclusion and 15 nodes. Both methods predicted a final time of approximately $58 \mathrm{~s}$.
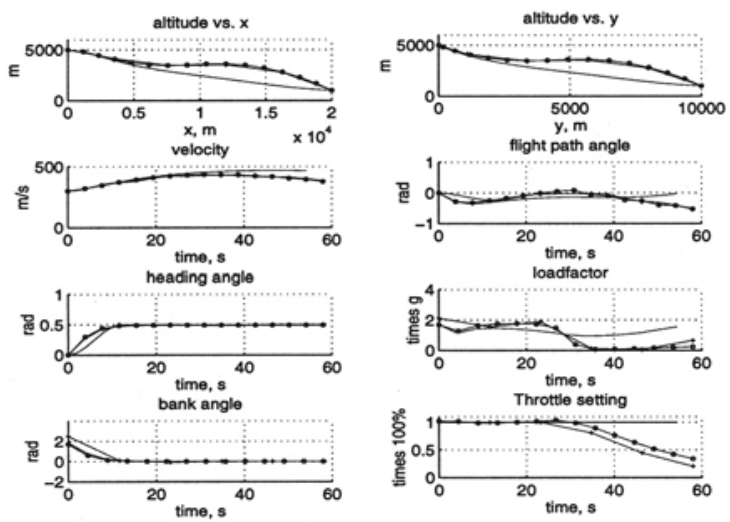

Figure 3 The state and control variable histories of the descent. ' $t$ ' corresponds to collocation and ' $O$ ' to differential inclusion. The dashed line indicates the unconstrained solution. The control variables corresponding to the differential inclusion solutions have been calculated afterwards.

\section{CONCLUSIONS}

We have described two discretization schemes for direct trajectory optimization. The presented numerical examples suggest that both methods are capable of approximating optimal trajectories. The equidistant discretization scheme used in the examples is simple to implement but by no means the most efficient one. The accuracy can be further improved by adaptively redistributing the discretization nodes.

Both schemes converge robustly in the case of regular controls. Singular controls affect the convergence of the collocation method, but do not considerably influence the performance of the differential inclusion scheme. This is consistent with previous results (see 
Seywald (1994)). In addition, the differential inclusion scheme describes the system with smaller number of decision variables and constraints than collocation.

It should be noted that the methods do not provide any explicit information on the structure of the solution. The activity of state and control constraints can be deduced from the solution data, but singular control intervals cannot be identified without deriving the necessary conditions. On the other hand, these approaches do not require the optimal switching structure in advance, which is the case with indirect methods.

\section{REFERENCES}

Aubin, J-P. and Frankowska, H. (1989) Set-valued Analysis. Birkhäuser.

Betts, J. and Huffman, W. (1993) Path-Constrained Trajectory Optimization Using Sparse Sequential Quadratic Programming. Journal of Guidance, Control and Dynam$i c s, 16,1$.

Ehtamo, H.K., Raivio, T. and Hämäläinen, R.P. (1994) A method to generate trajectories for minimum time climb, in Preprints of the 6th international symposium on differential games and applications, St. Jovite, Canada.

Hargraves, C., Johnson, F., Paris, S. and Rettie, I. (1981) Numerical Computation of Optimal Athmospheric Trajectories. Journal of Guidance, Control and Dynamics, $4,4$.

Hargraves, C. and Paris, S. (1987) Direct Trajectory Optimization Using Nonlinear Programming and Collocation. Journal of Guidance, Control and Dynamics, $10,4$.

Lachner, R., Breitner, M., and Pesch, H.J. (1994) Three-Dimensional Air Combat: Numerical Solution of Complex Differential Games, in Preprints of the 6th international symposium on differential games and applications, St. Jovite, Canada.

Lee, E.B. and Markus, L. (1986) Foundations of optimal control theory. Wiley, New York.

Miele, A. (1962) Flight Mechanics. Addison-Wesley, Massachusetts.

Seywald, H. (1994) Trajectory Optimization Based on Differential Inclusion. Journal of Guidance, Control and Dynamics, 17, 3.

Seywald, H. Cliff, E. and Well, K. (1994) Range Optimal Trajectories for an Aircraft Flying in the Vertical Plane. Journal of Guidance, Control and Dynamics, 17, 2. 